\title{
Population Based Survey of Glucose-6-Phosphate Dehydrogenase (G6PD) Deficiency among People Living in Terai Districts of Nepal
}

\author{
Niraj Lamichhane ${ }^{1}$, Nabaraj Adhikari ${ }^{2}$, Upendra Thapa Shrestha $^{2}$, Komal Raj Rijal $^{2}$, Megha Raj \\ Banjara $^{2}$, Prakash Ghimire ${ }^{2 *}$ \\ ${ }^{1}$ Department of Microbiology, Kantipur College of Medical Science, Sitapaila, Kathmandu \\ ${ }^{2}$ Central Department of Microbiology, Tribhuvan University, Kirtipur, Kathmandu
}

*Corresponding author: Dr. Prakash Ghimire; Central Department of Microbiology, Tribhuvan University, Kirtipur, Kathmandu; Email: prakash.ghimire@microbiotu.edu.np

\begin{abstract}
Objective: This study was carried out to determine the prevalence of Glucose-6-phosphate dehydrogenase (G6PD) deficiency among population of selected malaria endemic districts in central and eastern terai of Nepal.
\end{abstract}

Methods: Six hundred seventy whole blood samples were collected from the indigenous peoples community, identified based on district public health office records for G6PDd in the past from Jhapa, Morang and Dhanusha districts endemic to malaria, during April to June 2013. Collected blood samples were tested on the sites by using BinaxNow G6PD test kit and CareStart ${ }^{\mathrm{TM}}$ G6PD test kits.

Results: The G6PD deficiency was found to be in $6.1 \%$ and $6.3 \%$ in BinaxNow and CareStart ${ }^{\mathrm{TM}}$ respectively. In 42 G6PD deficient cases number of male to female ratio was almost equal. Higher proportions of deficient cases were from Rajbanshi and Santhal communities than others. Highest number of deficient cases was in Jhapa followed by Morang and Dhanusha districts respectively.

Conclusion: G6PD deficiency in indigenous population group in estern and central terai are heterogenous. So the testing of G6PD before initiation of radical treatment fo Plasmodium vivax infection would be important for reduing the risk of hemolysis following Primaquine (PQ) administration. Rational evidence based PQ administration may be helpful in contributing towards the elimination of malaria from the country.

Key words: G6PD, BinaxNow G6PD and CareStart ${ }^{\mathrm{TM}}$ G6PD

\section{INTRODUCTION}

Glucose-6-phosphaste dehydrogenase (G6PD) is an important enzyme in cellular metabolism in the first and rate-limiting step of pentose-phosphate pathway. Among the functions of this pathway is the protection of cells from oxidative stress, through its role in conversion of NADP to NADPH, thereby replenishing the levels of reduced glutathione. As erythrocytes lack other detoxifying enzymes, people with G6PD deficiency are susceptible to oxidative stress in their red blood cells (Cappellini and Fiorelli 2008). The G6PD gene is located on the $X$ chromosome, and as a result deficiency shows $X$-linked inheritance, whereby a higher proportion of males suffer from the deficiency.

Malaria in humans is predominantly caused by two Plasmodium species, Plasmodium falciparum and Plasmodium vivax and is estimated to be spread over more than 90 countries, putting nearly 3.3 billion people at risk of disease (Guerra et al. 2010, WHO 2012). The prevalence of G6PD across countries was found to have a good correlation with those where, historically, malaria transmission has occurred (Howes et al. 2012). The explanation for this association has been that G6PD is associated with protection against $P$. falciparum (Mockenhaupt et al. 2003; Clark et al. 2009) and P. vivax infections (Leslie et al. 2010; Santana et al. 2013). The mechanism conferring resistance in G6PD subjects may be related to an impaired antioxidant defence in ring-stage parasitized red cells, which could lead to membrane damage, triggering increased removal of infected cells by phagocytosis before parasite maturation to the trophozoite and schizont stages (Ruwende et al. 1995). G6PD is also thought to be a protection factor against severe manifestations of malaria, although studies regarding which individuals, hemizygous males or heterozygous females, may be protected present 
discrepancies (Ruwende et al. 1995; Guindo et al. 2007). G6PD deficiency is common in malaria endemic regions and is estimated to affect more than 400 million people worldwide. It is a genetic defect, which is one of the most prevalent polymorphisms and enzymopathies in humans, particularly in males.

This genetic defect was discovered in 1956 when some patients developed haemolyticanaemia after the dose of the anti-malarial drug primaquine. G6PD-deficient erythrocytes are more susceptible to destruction by oxidative stress than normal erythrocytes due to the lower NADPH levels. Individuals with this genetic defect may exhibit non-immune haemolyticanaemia in response to a number of stimuli, most commonly infections or exposure to certain medications or chemicals. Fortunately, the large majority of G6PD deficient subjects have no clinical manifestations and the condition remains asymptomatic until they are exposed to a hemolytic trigger.

The geographical distribution of malaria is remarkably similar to the world distribution of deficient G6PD variants. It is postulated that the high frequency of G6PD deficiency has arisen because G6PD deficient variants confer some protection or resistance against malaria caused by Plasmodium falciparum and Plasmodium vivax.

In Southeast Asia, a large number of G6PD deficient variants have been reported from various populations. In Nepal, previous studies have shown that G6PD deficiency in Tharu ethnic inhabitants of Chitwan districts (WHO 2013). With the development after 1960 's and movement of population the scenario is likely to be changed. In a study done by Suzuki et al. 2007 it was observed that the group that has lived for many decades in malaria endemic lowland area, the Danuwar, was found to have a high prevalence of alpha-thalassaemia (79.4\%) and low prevalence of haemoglobin E and G6PD deficiency. Much lower prevalence of alpha-thalassaemia were observed in the Newar $(20.5 \%)$, Parbate $(16.5 \%)$ and Tamang $(8.8 \%)$, who, until the 1950s, all spent their hot-season nights in malaria-free areas at higher altitude.

Glucose-6-phosphate dehydrogenase deficiency poses a significant impediment to primaquine use for the elimination of liver stage infection with Plasmodium vivax and for gametocyte clearance, because of the risk of life-threatening haemolytic anaemia that can occur in G6PD deficient patients. Although a range of methods for screening G6PD deficiency have been described, almost all require skilled personnel, expensive laboratory equipment, freshly collected blood, and is time consuming; factors that render them unsuitable for mass-screening purposes. So commercially available RDT/ICT card tests: BinaxNow- G6PD and CareStart G6PD were used for determination of G6PD deficiency for mass screening purposes (Tinley et al. 2010, Kim et al. 2011, vonFricken et al. 2014). This cross sectional study was designed to detect the prevalence of G6PD deficiency in indigenous peoples communities of Jhapa, Morang and Dhanusha districts. Evidence from such a study would be important for taking policy decision on the use of G6PD RDTs, and use/abstinence of Primaquine in specific population/age groups, as a radical treatment of malaria.

\section{MATERIALS AND METHODS}

Study design, study site and study population: The study was designed as a descriptive cross- sectional study. The study was conducted during April to June 2013. The study sites were malaria endemic village development communities (VDCs) of Jhapa, Morang and Dhanusha districts which are in moderate malaria risk identified by the last 5 years malaria data and the recent micro-stratification study report (EDCD/DoHS/ $\mathrm{MOH}$ Govt. of Nepal. Micro-stratification of malaria risk in Nepal 2013). Information as per the questionnaire were obtained from 670 volunteers recorded in the case record format and analyzed following statistical tools. A total of 670 blood samples were collected from volunteers representing different ethinic groups in the age groups of 5-64 years and agreeing to partiucipate in the study from Jhapa, Morang and Dhanusha districts. (NHRC ERB Reference no. 1134, 23 March 2013) and tested qualitatively for detecting G6PD deficiency using both BinaxNow G6PD and CareStart ${ }^{\mathrm{TM}}$ G6PD rapid diagnostic test kits by WHO Nepal for the survey.

Inclusion and exclusion criteria: Any person in the age group 5-64 years living in the malaria endemica area of above 3 districts were included. Any person temporarily staying in the area, however not from the indigenous ethnic groups and pregnant women or children below 5 years, and older persons above 64 years were excluded from the study.

Sample collection and testing: The blood samples from the voluteers were taken by squeezing fingertip 
and piercing with lancet. The qualitative G6PD testing were performed using BinaxNow G6PD and CareStart $^{\mathrm{TM}}$ G6PD test kits. All the tests were done at the collection site, maintaining aseptic condtion and universal precations.

Testing of G6PD deficiency by BinaxNow kit: BinaxNow G6PD test kits were used for follwoing manufacturer's instructions. Briefly, the device from the pouch was removed prior to use, brought to room temperature, and was recorded on the front of the device. The test was performed at the temperature between $18^{\circ} \mathrm{C}$ to $25^{\circ} \mathrm{C} .70 \mu \mathrm{l}$ of reagent- $\mathrm{A}$ and $10 \mu 1$ of blood from the volunteer was mixed in the sample preparation vial with the help of sterile micropipette mixed 3 times with push and pull by micropipette. Fifty microliter of mixed blood sample was added slowly to the middle of white pad of the test. The adhesive stripe was removed and the device was closed. The result was noted after 7 minutes. A distinct color change to black/brown on the top half of the reaction pad in visible reading window was considered normal while if there was no color change in the top half of the reaction pad in 7 minutes the samples were considered as G6PD deficient (Tinley et al. 2010)

Testing of G6PD deficiency by CareStart ${ }^{\mathrm{TM}}$ kit method: CareStart $^{\mathrm{TM}}$ G6PD testing was performed following manufacturers' instruction. Briefly, the area of fingertip to be pierced was cleaned with alcohol pad and fingertip was squeezed and pierced using sterile lancet. Blood sample $(2 \mu \mathrm{l})$ was collected using sample pipette provided by the kit. Whole blood $(2 \mu \mathrm{l})$ was applied to the sample well in the test kit. Immediately after application of blood 2 drop (100 $\mu$ l) of assay buffer was added to buffer well. The result was noted 10 minutes. Samples with normal G6PD produced a distinct purple color in the result window, ehile no color change was observed with G6PD deficient samples (Kim et al. 2011).

\section{RESULTS}

During the study period, a total of 670 whole blood samples were tested by rapid screening test for G6PD (Glucose-6-phosphate dehydrogenase) deficiency in human blood by using Carestart ${ }^{\mathrm{TM}}$ G6PD kit and BinaxNow G6PD kit.

\section{Comparison of G6PD diagnostic test kits}

Out of 670 blood samples tested for G6PD deficiency, $6.1 \%$ (41) were found to be G6PD deficient in BinaxNow while $6.3 \%$ (42) cases were found G6PD deficient in CareStart $^{\mathrm{TM}}$. G6PD deficiency detected by test kit only in 30 samples. Of the total 41 G6PDd detected samples by BinaxNow kit, 11 were found by CareStart ${ }^{\mathrm{TM}}$ kit. Similarly, of the total 42 G6PDd samples detected by CareStart ${ }^{\mathrm{TM}}$ kit, 12 were found by BinaxNow kit. As both of the test kits are competing RDTs and we didnot have any gold standard to compare either of both. We only could compare the easiness of testing kits, visibility of the test results in the kits and not the sensitivity and specificity of the tests for comparison. The detail of comparison is as below (Table 1).

Table 1: Comparison between BinaxNow and CareStart ${ }^{\mathrm{TM}}$ kit

\begin{tabular}{|c|c|c|c|c|}
\hline \multirow{2}{*}{\multicolumn{2}{|c|}{ Test kits }} & \multicolumn{2}{|c|}{ BinaxNow G6PD kit } & \multirow{2}{*}{ Total } \\
\hline & & Deficient & Normal & \\
\hline \multirow{2}{*}{ CareStart $^{T M}$ Kit } & Deficient & 30 & 12 & $42(6.3)$ \\
\hline & Normal & 11 & 617 & $628(93.7)$ \\
\hline Total & & $41(6.1)$ & 629 (93.9) & 670 \\
\hline
\end{tabular}

Sex wise distribution of G6PD deficient cases Among 42 G6PD deficient cases 21(7.3\%) were male and
$21(5.4 \%)$ were female. There is no significant association of G6PD deficiency with sex ( $p=0.529$ ) (Table 2).

Table 2: Sex wise distribution of G6PD deficient cases

\begin{tabular}{lccc}
\hline Sex & No. of G6PDd & Percent of G6PDd & p-value \\
\hline Male $(n=285)$ & 21 & 7.3 & 0.529 \\
Female $(n=385)$ & 21 & 5.4 & \\
\hline
\end{tabular}

Distribution of G6PD deficient cases in different ethnic groups

G6PD deficiency was evaluated in the ethnic group population of the above 3 districts. The highest percentage of deficient cases were from Rajbanshi followed by Santhal, Rishidev and Tharu community among the three districts (Table 3). 
Table 3: Distribution of G6PD deficient cases in different ethnic groups

\begin{tabular}{lcc}
\hline Ethnic group & Total test cases & Deficient cases (\%) \\
\hline Tharu & 151 & $8(5.3)$ \\
Rajbanshi & 154 & $18(11.7)$ \\
Mushahar & 204 & $7(3.4)$ \\
Rishidev & 61 & $4(6.6)$ \\
Santhal & 49 & $5(10.2)$ \\
Others (non-ethnic) & 51 & $0(0)$ \\
\hline
\end{tabular}

District wise distribution of G6PD deficient cases

Highest percentage of deficient cases was noted from Jhapa district followed by Morang and Dhanusha

Table 4: Region wise distribution of G6PD deficient cases

\begin{tabular}{lcc}
\hline District & Total number of test cases & Confirmed deficient cases (\%) \\
\hline Jhapa & 224 & $22(9.8)$ \\
Morang & 225 & $13(5.8)$ \\
Dhanusha & 221 & $7(3.1)$ \\
\hline
\end{tabular}

\section{DISCUSSION}

Over one third of world's population lives at the risk of Plasmodium vivax infection. Limited evidence underpins estimation of clinical cases, however globally about 400 million clincal cases are reported anually including potentially severe illness and death. In the context of malaria elimination, therapy must target all infections including asymptomatic and sub-microscopic blood stage infections, dormant liver stage hypnozoites as well as clinical cases. One of the many consequences of neglect for last half century of $P$. vivax has been the failure to address the Primaquine toxicity problem with G6PD. No non-toxic therapeutic alternatives exist, and existing G6PDd diagnostics are largely impractical in point of care settings (Ghimire et al. 2017).

Glucose-6-phosphate dehydrogenase (G6PD) is a ubiquitously expressed enzyme that has a housekeeping role in all cells, and is particularly critical to the integrity and functioning of red blood cells (RBCs). G6PD is an important enzyme in cellular metabolism and ratelimiting step of pentose-phosphate pathway. Among the functions of this pathway is the protection of cells from oxidative stress, through its role in conversion of NADP to NADPH, thereby replenishing the levels of reduced glutathione. As erythrocytes lack other detoxifying enzymes, people with G6PD deficiency are susceptible to oxidative stress in their red blood cells (Cappellini and Fiorelli 2008).

Primaquine is the only-antimalarial drug recommended by WHO for the radical cure of malaria. However, a major barrier to widescale adoption of both of these drugs is toxicity in people with G6PD deficiency. While
respectively.There was significant association between G6PD deficiency and region $(p=0.010)$.

all people exposed to primaquine experience some drop in haemoglobin concentrations (Kellermeyer et al. 1962), people with G6PD deficiency are more likely to experience severe haemolysis, leading to severe haemolyticanaemia and, potentially, death.

The blood samples from the volunteer participants inhabiting the malaria endemic districts and consenting to participate in the study through the written consent were tested on the spot by using G6PD kits BinaxNow G6PD and CareStart ${ }^{\mathrm{TM}}$ G6PD kits. Out of 670 volunteer tested for G6PDd, 6.1\% were detected as G6PD deficient were detected by BinaxNow G6PD RDT and $6.3 \%$ by CareStart ${ }^{\mathrm{TM}}$ G6PD RDT. Although the detection of G6PDd by BinaxNow is slighly more than that by CareStart ${ }^{\mathrm{TM}}$ RDT the difference is not statistically significant. Low prevalence of G6PD deficiency was reported from Latin America (Monteiro et al. 2014) whereas average prevalence of G6PD deficiency $(2.1 \%)$ with apparent differences between ethnic groups and geographical regions was reported from Tanjania (Rebholtz et al. 2006). The variation is due to the geographical variation in sampling area, and ethnic groups of the people.

Compared to BinaxNow G6PD kit method sensitivity and specificity of the Carestart ${ }^{\mathrm{TM}}$ is $73.17 \%$ and $98.09 \%$. Positive predictive value of test is $71.42 \%$ and negative predictive value is $98.24 \%$. However, different sensitivity (86\%) and nearly same specificity (98\%) value were reported from a study done in UK (Tinley et al. 2010). The result was also inconsistence to the similar study in cape coast Ghana (Daniel et al. 2010). The factors responsible for the poor performance of the 
kits might be the storage temperature, the temperature at which the test is performed, the ethnic community of Ghana and Nepal and also the geographical distribution of Nepal.

Among the G6PD deficient cases, male and female ratio was 1:1 and there was no significant relationship between gender and occurrence of G6PD deficiency $(p=0.529)$. This finding is different than other studies in which number of G6PD deficient cases were more in male (Nimol et al. 2010; Julien et al. 2011). The reason for this is still unknown and requires further molecualr level investigation with more countrywide sample representation.

Looking at G6PDd distribution in different indigenous groups staying in the study area highest number of confirmed deficient cases were from Rajbanshi (11.7\%) followed by Santhal (10.2\%), Rishidev (6.6\%) and Tharu $(5.3 \%)$ in the three study districts. Higher prevalence of G6PDd was found in Jhapa (9.8\%) and Morang districts $(5 ; 8 \%)$ this result is concordance with previous studies of Hongkong, Combodia, UK and in Ghana (Nimol et al. 2010; Daniel et al. 2012). The parasite causing diseases like malaria cannot survive well in the G6PD deficient cells. One of the schools of thought is "The ethnic community or inhabitants of malaria endemic areas are being exposed to such parasites for centuries and hence deficiency is developed and inherited for generations for providing protection against deadly $P$. falciparum malaria. In context of Nepal, Tharu, Chaudhary, Rajbanshi, Santhal and Rishidev communities are majority inhabitants of malaria endemic districts since centuries, are still thought to be immune for deadly falciparum malaria. The G6PD deficiency on such community might have developed in the long run to maintain their immunity against $P$. falciparum malaria, The scientific basis of which requires further studies at molecualr level. This study simply tried to find out the qualitative information on G6PDd in different indigenous population taking in to consideration for introducing mandatory G6PDd testing in confirmed $P$. vivax cases, for effective implementation of radical treatment with Primaquine following Chloroquine as per national malaria treatment protocol.

Malaria elimination will be possible only with serious national, regional and international efforts addressing asymptomatic infection and persistent $P$. vivax infection. Currently available drugs that can radically cure $P$. vivax malaria and are able to reduce transmission of malaria parasites are those in the 8-aminoquinoline family, such as PQ. Tafenoquine, which is also an 8-aminoquinoline, is currently being assessed as a single-dose radical cure therapy, once it is approved for marketting following successful complition of clinical trials and market authorization. Unfortunately, individuals carrying the G6PDd are at risk to develop severe haemolysis if exposed to these drugs, implying that radical cure regimens will require broader testing for G6PD deficiency.

\section{CONCLUSION}

The higher prevalence of G6PDd in certain ethnic group; Rajbanshi, Santhal, Rishidev and Tharu in comparison to Brahmin and Chhetri in the eastern region clearly demonstrates the importance of G6PD deficiency screening prior to Primaquine prescription for the treatment of vivax malaria in the malaria endemic areas. Knowing the G6PDd status gives leverage to use 14 days primaquine in G6PD normal patients, while weekly primaquine under close clinical monitoring/medical supervision with ready access to blood transfusion services in G6PDd cases. In absence of appropriate G6PDd testing facility and lack of knowledge on prevalent genotype and severity, it will be prudent to err on the safe side. Rational use of such radical treatment facilitates the country in malaria elimination as planned by NMSP-2016.

\section{ACKNOWLEDGEMENTS}

The authors express sincere thanks to WHO Nepal for providing the financial support to the study, EDCD, DPHO for thier cordination and voluteers who provided consent and samples for testing.

\section{REFERENCES}

Cappellini MD and Fiorelli G (2008) Glucose-6phosphate dehydrogenase deficiency. Lancet 371(9606): 64-74.

Clark TG, Fry AE, Auburn S, Campino S, Diakite M, Green A, Richardson A, Teo YY, Small K, Wilson J, Jallow M, Sisay-Joof F, Pinder M, Sabeti P, Kwiatkowski DP and Rockett KA (2009) Allelic heterogeneity of G6PD deficiency in West Africa and severe malaria susceptibility. Eur J Hum Genet 17: 1080-1085.

Daniel A, Mensa S and Bonsu A (2012-2013) Molecular basis of glucose 6 phosphate dehhydrogenase deficiency in Cape coast Ghana. Department of Biotechnology and Biochemistry Kwame 
Nkrumah University Ghana.

Epidemiology and Diseases Control Division, DoHS/ $\mathrm{MOH}$ Govt of Nepal. Micro-stratification of malaria risk in Nepal-Study reprot -2013.

Ghimire P, Singh N, Ortega L, Rijal KR, Adhikari B, Thakur GD and Marasini B (2017) Glucose-6-phosphate dehydrogenase deficiency in people living in malaria endemic districts of Nepal. Malar J 16: 214.

Guerra CA, Howes RE, Patil AP, Gething PW, Van Boeckel TP, Temperley WH, Kabaria CW, Tatem AJ, Manh BH, Elyazar IRF, Baird JK, Snow RW, Hay SI (2010) The international limits and population at risk of Plasmodium vivax transmission in 2009. PLoS Negl Trop Dis 4: e774.

Guindo A, Fairhurst RM, Doumbo OK, Wellems TE and Diallo DA (2007) X-linked G6PD deficiency protects hemizygous males, but not heterozygous females against severe malaria. PLoS Med 4: e66.

Howes RE, Piel FB, Patil AP, Nyangiri OA, Gething PW, Dewi M, Hogg MM, Battle KE, Padilla CD, Baird JK and Hay SI (2012) G6PD deficiency prevalence and estimates of affected populations in malaria endemic countries: a geostatistical model-based map. PLoS Med 9: e1001339.

Julien D, Moutawakilou G and Josheph A (2010) Prevalance of glucose 6 phosphate dehydrogenase deficiency among infants and childrens of parakou Benin. Faculty of Medicine. Parakou Benin. 0971-9032.

Kellermeyer RW, Tarlov AR, Brewer GJ, Carson PE and Alving AS (1962) Hemolytic effectof therapeutic drugs: clinical considerations of the primaquinetypehemolysis. JAMA 180: 388-394.

Kim S, Nguon C, Guillard B, Duong S, Chy S, Sum S, NhemS, Bouchier C, Tichit M, Christophel E, Taylor WRJ, Baird JK and Menard D (2011) Performance of the CareStartTM G6PD deficiency screening test, a point-of-care diagnostic for primaquine therapy screening. PLOS ONE 6: e28357.

Leslie T, Briceño M, Mayan I, Mohammed N, Klinkenberg E, Sibley CH, Whitty CJM and Rowland M (2010) The impact of phenotypic and genotypic G6PD deficiency on risk of Plasmodium vivax infection: a case-control study amongst Afghan refugees in Pakistan. PLoS Med 7: e1000283.

Mockenhaupt FP, Mandelkow J, Till H,Ehrhardt S, Eggelte TA and Bienzle U (2003) Reduced prevalence of Plasmodium falciparum infection and of concomitant anaemia in pregnant women with heterozygous G6PD deficiency. Trop Med Int Health 8: 118-124.

Monteiro MW,Val FA, Siqueira AM, Gabriel P, Franca GP, Sampaio VS, Melo GC, Almeida ACG, Brito MA, Peixoto HM, Fuller D, Bassat.Romero GS, Oliveira MF and Lacerda MG (2014) G6PD deficiency in Latin America: systematic review on prevalence and variants. MemInst Oswaldo Cruz, Rio de Janeiro, Vol. 109(5): 553-568.

Nimol K, Christophe B and Saorin K (2010-2012) Deficiency in Plasmodium falciparum and Plasmodium vivax malaria infected Cambodian patients.

Rebholz CE, Michel AJ, Maselli DA, Saipphudin K and Wyss K (2006) Frequency of malaria and glucose-6-phosphate dehydrogenase deficiency in Tajikistan. Malaria Journal 5: 51.

Ruwende C, Khoo SC, Snow RW, Yates SN, Kwiatkowski D, Gupta S, Warn P, Allsopp CE, Gilbert SC and Peschu N (1995) Natural selection of hemi and heterozygotes for G6PD deficiency in Africa by resistance to severe malaria. Nature 376: 246-249.

Santana MS, Monteiro WM, Siqueira AM, Costa MF, Sampaio V, Lacerda MV and Alecrim MG (2013) Glucose-6-phosphate dehydrogenase deficient variants are associated with reduced susceptibility to malaria in the Brazilian Amazon. Trans $R$ Soc Trop Med Hyg 107: 301-306.

Tinley KE, LoughlinAM, Jepson A and Barnett ED (2010) Evaluation of a rapid qualitative enzyme chromatographic test for glucose-6-phosphate dehydrogenase deficiency. Am J Trop Med Hyg 82: 210-214.

Von Fricken ME, Weppelmann TA, Eaton WT, Masse R, Madsen VE, Rochars B and Okech BA (2014) Performance of the CareStart glucose-6-phosphate dehydrogenase (G6PD) rapid diagnostic test in Gressier, Haiti.Am J Trop Med Hyg91: 77-80.

WHO-World Health Organization (2013) World Malaria Report 2013 Available from: who.int/malaria/ publications/world_malaria_report_2013/en/.

WHO-World Health Organization (2012) World Malaria Report 2012. Available from: who.int/malaria/ publications/world_malaria_report_2012/en/. 